\title{
Covid-19 - is it less severe in asthmatic and allergic children?
}

\author{
Chandra Sekhar Devulapalli ${ }^{1}$ \\ ${ }^{1} \mathrm{NAV}$
}

May 13, 2020

\begin{abstract}
It has been widely reported that vast majority of children have experienced only mild symptoms in the ongoing COVID-19 pandemic outbreak. It also appears that children have a minimal risk of developing COVID-19 and also minimal risk of a fatal outcome. Reasons for this is largely unknown in the present circumstances. Patients with severe and uncontrolled asthma have also been classified to be at increased risk of developing more severe COVID-19. It has been earlier speculated that children were less sensitive to COVID-19 because the maturity and binding ability of angiotensin-converting enzyme-2 (ACE2) in children may be lower than in adults. A recent study indicate that children with asthma and allergies have reduced ACE2 gene expression due to down-regulation of the ACE2 receptor. It is conceivable that virus-induced immune response and subsequent tissue damage may be less pronounced in children. So far, there is no evidence to suggest that children with chronic diseases like asthma and allergy will have a more severe reaction to COVID-19.
\end{abstract}

\section{Dear Editor,}

It is interesting to note that type 2 alveolar epithelial cells (AECII) which are primarily responsible for injury repair, regeneration and homeostatic maintenance probably have a important role in defining the severity of coronavirus disease 2019 (COVID-19) in children (1). The novel virus that causes severe acute respiratory syndrome coronavirus 2 (Sars-CoV-2) uses angiotensin-converting enzyme-2 (ACE2) as its cellular receptor facilitating virus entry and replication. As suggested in this commentary (1), injury and depletion of AECII which express ACE2 receptor may be directly related to severity and mortality due to COVID-19.

Children of all ages were sensitive to COVID-19 (2). It is still a mystery why most of the children's COVID19 cases were milder in clinical presentation $(3,4)$. It has been earlier speculated that children were less sensitive to COVID-19 because the maturity and binding ability of ACE2 in children may be lower than in adults. Probably the answer lies in ACE2 gene expression. Studies investigating factors affecting ACE2 gene expression have revealed that its upregulation is associated with smoking, overweight, diabetes and hypertension, all associated with increased severity of COVID-19.

Patients with severe and uncontrolled asthma have also been classified to be at increased risk of developing more severe COVID-19 according to Centre for Disease Control and Prevention. However, studies show that ACE2 is involved in protective mechanisms of the lung. It may protect against severe lung injury induced by respiratory virus infection in an experimental mouse model and in pediatric patients. ACE2 also protects against severe acute lung injury that can be triggered by sepsis, acid aspiration, SARS, and lethal avian influenza A H5N1 virus infection.

Results from an American study indicate that children with asthma and allergies have reduced ACE2 gene expression due to down-regulation of the ACE2 receptor (5). In this cohort study, ACE2 expression in respiratory cells was lowest in those with both high levels of allergic sensitization and asthma (5). This might explain why children with asthma and allergic sensitization have apparently less serious illness. However, 
further investigations on factors beyond ACE2 expression are necessary to provide insights into COVID-19 disease pathogenesis. At present, reduced ACE2 expression appears to be a potential "protective" mechanism of reduced covid-19 severity in patients with asthma and respiratory allergy.

CHANDRA SEKHAR DEVULAPALLI M.D., Ph.D.

Senior Medical Consultant and Pediatrician

Norwegian Labour and Welfare Administration (NAV), Advisory Medical Service, work and benefits Kristiania, Oslo, Norway.

chandev@gmail.com

Conflicts of interest: None.

Finance: None.

\section{References}

1. Im, DD. Why is Coronavirus Disease 2019 not as severe in children?-A look at type 2 alveolar cells. Pediatric Pulmonology. 2020; 55: 1332-1333.https://doi.org/10.1002/ppul.24786

2. Dong Y, Mo X, Hu Y, et al. Epidemiology of COVID-19 Among Children in China. Pediatrics. 2020;e20200702. doi:10.1542/peds.2020-0702.

3. Lee PI, Hu YL, Chen PY et al. Are children less susceptible to COVID-19? J Microbiol Immunol Infect. 2020;S1684-1182(20)30039-6. doi:10.1016/j.jmii.2020.02.011

4. Devulapalli CS. Covid-19 - a mild disease in children. Tidsskr Nor Laegeforen. 2020 Mar 24;140(6). doi: 10.4045/tidsskr.20.0231. Print 2020 Apr 21.

5. Jackson DJ, Buses WW, Bacharier LB et al. Association of Respiratory Allergy, Asthma and Expression of the SARS-CoV-2 Receptor, ACE2. Journal of Allergy and Clinical Immunology (2020).https://doi.org/10.1016/j.jaci.2020.04.009 